\title{
Research on Creating Ecological Highway for Serving Construction of Powerful Transportation Country
}

\author{
Anqi $\mathrm{Lv}^{1}$, Dong Xin ${ }^{2 *}$, Lu Zhang ${ }^{3}$, Ling Shi ${ }^{4}$ and Rui Geng ${ }^{5}$ \\ ${ }^{1}$ Research Institute of Highway Ministry of Transport / Zhonglu Gongke (Beijing) Investment \& Consulting Co., Ltd., Beijing,100088, \\ China \\ ${ }^{2}$ Jiaoke Transport Consultants Ltd., Beijing, 100191, China \\ ${ }^{3}$ Research Institute of Highway Ministry of Transport, Beijing, 100088, China \\ ${ }^{4}$ Research Institute of Highway Ministry of Transport / Zhonglu Gongke (Beijing) Investment \& Consulting Co., Ltd., Beijing, 100088, \\ China \\ ${ }^{5}$ Research Institute of Highway Ministry of Transport, Beijing ,100088, China
}

\begin{abstract}
Green transportation is an important feature and intrinsic requirement of a powerful transportation country. Therefore, we shall fully implement the spirit of the 19th National Congress of the Communist Party of China, effectively implement the new development concept, further promote the development of green transportation and serve the construction of a powerful transportation country. An important part of green transportation system is the ecological highway. Therefore, this paper firstly clarifies the concept, connotation and characteristics of ecological highway, defines the whole life cycle of ecological highway construction, that is, different control points in each stage from planning, design, construction to operation, management and maintenance, introduces the built ecological highway in China, proposes the implementation scheme of ecological highway construction based on the specific requirements and key technologies and finally elaborates the safeguard measures for ecological highway construction. Besides, this paper also provides a reference for clarifying the idea and development of ecological highway construction.
\end{abstract}

\section{Introduction}

The spirit of the 18th National Congress of the Communist Party of China defines to promote the new development of socialism with Chinese characteristics in the overall layout of "five-in-one", and the ecological civilization construction is one of the "five-in-one" layout. Meanwhile, we shall also follow the strategic requirements of ecological civilization construction during transportation construction and development, and put forward the new construction idea of "ecological highway" during ecological civilization construction.

\section{Basic theoretical research of ecological highway}

\subsection{Concept of ecological highway}

Ecological highway refers the builder considers the whole process of decision-making, design, construction, operation and management comprehensively based on the benign cycle of ecosystem (nature), combines the nature, human and highway organically, integrates them into ecological design method, prevent development and construction at the expense of ecological resources, not only considers the interaction between human activities and highway, but also pay attention to maintain the harmony between the human and living nature conditions, follows the natural development rules and forms a sustainable highway development mode with safe and comfortable driving, efficient and convenient transportation, complete and harmonious landscape and natural protection.

\subsection{Connotation of ecological highway}

On the macro level, ecological highway is a complex formed by the interaction, mutual influence and mutual restriction of various factors such as ecological environment, social economy and construction technology, it is the concrete embodiment of sustainable development strategy in the field of highway and compatible with the regional environmental carrying capacity.

On the micro level, ecological highway is a marginal ecological engineering and construction form that not only can obtain social and economic benefits, but also can promote ecological environment protection, with the ecological principle as the guidance and the ecological environment and natural conditions as the orientation.

$\overline{{ }^{*} \text { Corresponding author's e-mail: } 254836782 @ q q . c o m}$ 
On the implementation level, ecological highway refers to the integration with the natural environment in the design, construction and operation of highway to minimize the damage and pollution to the environment. It shall not only create a good artificial environment, but also deal with the relationship with the overall ecosystem to achieve the coordinated development with the environment. Therefore, such idea requires people to fully respect and correctly understand nature, reasonably and effectively use natural laws to build and manage highway, and minimize the damage of highway construction to nature.

\subsection{Characteristics of ecological highway}

The theoretical system of ecological highway no longer blindly separates the relationship between human and nature, but integrates human into the whole natural ecosystem. In order to realize the unified and comprehensive maximization of economic benefits, social benefits and environmental benefits of ecological highway, we shall consider the research object comprehensively in the "highway-nature-economy-society" compound system, and combine the ecological environment system with different nature with the highway economic system research organically.

\subsubsection{Good ecological environment}

The highway construction is restricted by the geological, topographical and hydrological conditions as well as the existing technical conditions such as the productivity level, production technology and production tools which will inevitably affect the ecological environment along the line, such as vegetation deterioration, soil erosion and land subdivision. During ecological highway construction, we shall restrict the damage of highway construction to the minimum range by taking various engineering measures, biological measures, agronomic measures and management measures comprehensively under the existing conditions. For the caused damage, we shall take the best recovery measures to rebuild a new ecosystem, enable the new community to achieve the top community and compensate the occupied land. At present, economic compensation measures are usually taken for the damage of natural resources caused by construction projects in China.

\subsubsection{Good landscape environment}

The characteristics of ecological highway on the landscape level are the most intuitive and easily perceived. The impression of ecological highway to travelers should not only be steel mesh, concrete wall and asphalt pavement, but also create an environment of "beautiful scenery around your while driving". The ecological highway shall make the route adapt to the landscape to the greatest extent by means of reasonable route selection and its characteristics. We shall show and strengthen the highway landscape with the layout and design and improve it with scientific landscaping, thereby bringing aesthetic feeling to travelers and maintaining the balance of natural ecosystem.

\subsubsection{Safety and high efficiency}

The ecological highway inevitably requires safe and comfortable driving and efficient and convenient transportation. Therefore, the ecological highway infrastructure shall create necessary conditions for the movement of goods flow, passenger flow, energy flow, information flow and value flow, and reduce the economic loss and pollution to the ecological environment along the highway while accelerating the sequential movement of various flows. The basic idea of ecological highway construction is to run through the concept of ecological highway construction, it is a systematic project and shall combine with the environment in the whole process of planning, design, construction and operation.

\subsection{Practical analysis of typical ecological highway in China}

\subsubsection{Chuanzhusi-Jiuzhaigou highway}

Chuanzhusi-Jiuzhaigou highway is the first comfortable and beautiful plateau ecological highway in China. Chuanzhusi-Jiuzhaigou highway is located in the northwest of Sichuan and belongs to a key construction project of the Ministry of Communications. Chuanzhusi-Jiuzhaigou highway is a mountain second-class highway with the total length of $94.14 \mathrm{~km}$ and was completed in September 2003. Since the project area is high in altitude, sparsely populated and vulnerable in ecological environment, ecological protection and restoration are the key ideas of the project. we have protected the ecological environment along the highway effectively by formulating a perfect ecological protection principle and implementing it during design and construction. The vegetation restoration rate in the area of highway has reached more than $90 \%$ by means of conventional greening technology and innovative ecological restoration technology, thereby creating a good tourism environment.

\subsubsection{Simao-Xiaomengyang highway}

Simao-Xiaomengyang highway is the first ecological highway passing through tropical rainforest in China Simao-Xiaomengyang highway is a mountainous four-lane highway with the total length of $97.75 \mathrm{~km}$ and was completed on April 6, 2006. In order to achieve the effective coordination between highway construction and environmental protection, the builders of Simao-Xiaomengyang highway puts forward the location of "protect, integrate, return to and enjoy nature" and the new concept of humanistic care construction. Therefore, Yunnan has taken the lead in implementing omnibearing and whole-process environment management system and 
the environmental protection guarantee system of "government supervision, owner management, enterprise control and whole staff implementation" are implemented in China.

\subsubsection{Yulin-Jingbian highway}

Yulin-Jingbian highway is the first desert ecological highway in China. The total length of Yulin-Jingbian highway is $116 \mathrm{~km}$, and it was completed formally in August, 2003. The builders made every endeavor to plant nearly 40,000 mu of desert shrubs along the highway for sand stabilization and road building, and built two high-standard tree belts (width: $500 \mathrm{~m}$ ) for wind prevention and sand fixation on both sides of highway. At present, the whole greening shelterbelt has been completed on both sides of the highway and become a "green corridor" in the desert

\section{Implementation scheme of ecological highway construction}

\subsection{General idea}

To construct the ecological highway, we shall improve the utilization of new materials, new equipment and new technology through technological and management innovation, so that the energy consumption, carbon dioxide and pollutant emissions of the highway in the whole life cycle can be significantly reduced and the environmental benefits can be significantly improved. In the whole process of planning and design, construction and construction, operation and management of ecological highway engineering, we shall make full use of advanced energy saving and emission reduction technology, reduce energy consumption and emission of the project, improve the resource use efficiency, advocate the recycling of renewable resources and fully embody the core concept of "environmental protection, recycling and low carbon" in the fields such as the subgrade and pavement engineering, bridge engineering, tunnel engineering, traffic safety facilities engineering, housing construction engineering, construction organization and management, operation management and maintenance.

\subsection{Functional requirements}

The most basic principle of ecological highway construction is people-oriented. Therefore, we put forward more, newer and higher specific functional requirements to ensure the highway engineering construction quality by means of the existing technologies.

\subsubsection{Driving safety under special geology, linear and weather conditions}

The driving safety of the highway is the most basic and essential requirement for the highway to provide users with high-quality service, and it is also the premise of all higher-level technical requirements (such as environmental protection highway, beautification highway or ecological highway). Safety is the most basic requirement in the existing highway engineering technical standards. The current technical standards in China can guarantee the driving safety under the general geological, linear and weather conditions, but we shall pay special attention to the driving safety under the special conditions and carry out the following research: (1) fog elimination research of plain or mountain expressway in spring and autumn; (2) research on thermal insulation pavement to prevent frost boiling of highway in permafrost, glacier or Tianshan No. 1 Glacier in the Qinghai-Tibet Plateau and severe winter area and guarantee driving safety; (3) research on snow and ice melting pavement in freezing rain or sleet area; (4) research on anti-sliding structure and lateral friction coefficient (high anti-sliding pavement) of large longitudinal slope and sharp turn road section in mountainous area under the weather such as rain, snow and freezing rain; (5) research on linear improvement of mountainous highway or permeable anti-sliding pavement of highway with poor linear topological restriction and the traffic engineering and safety measures.

\subsubsection{Reduction of driving noise and vibration}

The influence of driving noise and vibration on the production and life of the residents along the highway is becoming more and more serious. There have been many disputes between the residents and the highway transportation department. Therefore, it is necessary to study the following topics: (1) control on driving noise and vibration of highway; (2) research on low-noise pavement; (3) research on noise reduction and sound insulation engineering facilities; (4) technical research on measurement standard, detection and evaluation of the highway noise; (5) technical research on measurement standard, detection and evaluation of driving vibration; (6) research on pavement to reduce vibration. The research content of reducing noise and vibration of highway belongs to highway environmental protection. China has a good foundation noise and vibration reduction, but is still weak compared with developed countries. Therefore, we shall learn from the rich experience of developed countries in low-noise asphalt pavement, cement pavement and noise reduction and sound insulation engineering facilities.

\subsubsection{Reduction of tail gas pollution along the highway}

The core of reducing tail gas pollution is to gradually improve the vehicle exhaust emission standard of vehicle. What can be done in the highway engineering is to consider the need to absorb tail gas when selecting the tree species and grass for greening, that is, the need to absorb the harmful gas in tail gas shall also be considered except for achieving afforestation and beautification. In order to build an international metropolis, some small 
and medium-sized cities expand the pavement width to $50-80 \mathrm{~m}$ or even more than $100 \mathrm{~m}$. There is no greening on both sides and the center of the road. In summer, the strong sunlight will produce extremely high heat, which results in serious gas and other pollution. Therefore, this is a typical practice that only pays attention to engineering but ignores environmental protection and ecology. The research on reducing tail gas pollution also belongs to the category of environmental protection of highway. The restriction to highway exhaust emission standard is not only related to the traffic flow of this section of highway, but also related to the factors such as ventilation and the speed of exhaust gas dissipation.

\subsubsection{Reduction of light reflex and pollution of pavement}

Connotation of light pollution reduction: 1) reduce the eye reflex irritation of drivers and passengers; 2) light pollution of surrounding environment at night. The problem of light pollution is not easy to solve from the perspective of vehicles, and sufficient brightness and visibility shall be ensured when driving at night. At present, the application of light shield in our country is mainly to solve the glare effect of vehicle light in the opposite direction, and the light pollution of low-class highway can only be solved through light control. However, there is little research on the reflection and reflection intensity of strong sunlight on the highway at home and abroad. The current solution is to set up a light shield in the front row of the vehicle, which is assisted by the anti-sliding structure on the pavement, not only meeting the requirements of safe anti-sliding material, structure and surface texture, but also alleviating the reflection and reflection intensity of sunlight to a certain extent. The color of pavement is not only related to the requirements of skid resistance, light reflection, driving guidance and aesthetics, but also related to the speed of melting ice and snow on the pavement and the driving safety in icy and snowy days. The research on texture structure, pavement optics and chromatics of expressway and other highways shall be carried out simultaneously.

\subsubsection{Reduction of wastes of pavement construction and reconstruction project}

With the increase of mileage and quantity of highway construction in China, we are faced with the huge environmental pressure of waste residue and materials in the reconstruction of pavement engineering. Therefore, it is necessary to recycle the asphalt mixture, cement concrete pavement and base material. From the perspective of environmental protection, the pressure of cement pavement and water-stable base material on environmental protection is less than that of asphalt pavement. After cement hydration, the natural inorganic rock material-tobermorite will not be aging and has good compatibility with the environment. However, asphalt materials may cause pollution to both open drainage and groundwater, especially the digging and milling wastes from asphalt pavement, it shall not be discarded arbitrarily but shall be stacked and buried in a centralized way and recycled when the quantity is enough, so as to effectively protect the river environment, groundwater environment and soil environment.

\subsubsection{Reduction of destruction and interference on original ecological environment}

During ecological highway construction, we shall also try to minimize the interference to the original ecological environment, vegetation, trees, topography and landform. The specific methods include: reducing the height of subgrade and the amount of earthwork for filling and excavation as far as possible, protecting vegetation and trees, and reducing the amount of waste earth and stone. In addition, we shall green the vegetation of discarded places, try to make the soil slope gentle and reduce land erosion. The water flowing from side ditch into brook and river shall be clean and basically have no sediment. In the section where the slope cannot be slowed down, the upper and lower slopes shall be protected and afforested reliably; smooth blasting shall be used as much as possible for the rock slope, but shotcrete or full mortar rubble which can only meet the engineering support requirements shall not be used for protecting the broken rock slope, and the requirements for greening and beautification shall be considered; the viaduct shall be arched to reduce the height of approach subgrade, the earthwork quantity and the destruction to the original landform; the distance between the expressway and the first-class highway shall be widened in the mountainous area, especially for the steep valley or mountain stream, we can build a double-layer bridge on the river channel for the expressway and first-class highway, with water flowing under the bridge.

\subsection{Key technologies}

During ecological highway construction, we mainly focus on the processes and links such as planning, design, construction, maintenance, operation and management, developed more than 40 key technologies in green energy application, green service area, green construction technology application, smart highway, environmental protection and resource recycling and green transport capacity construction, and create the ecological highway in whole process, full range and all fields with new materials, technologies and processes of energy saving and emission reduction. The key technical essentials of ecological highway construction are shown as the figure below: 
Table 1 Key technical essentials list of ecological highway construction

\begin{tabular}{|c|c|}
\hline \multicolumn{2}{|c|}{ Key technical essentials of ecological highway construction } \\
\hline Indoor daylight illumination technology & Site LED energy-saving technology \\
\hline Solar energy hot-water system & Air energy hot-water system \\
\hline Sewage treatment & Rainwater resource technology \\
\hline Building wall heat-insulation technology & Restraint of energy-saving equipment purchasing \\
\hline Type selection design of key route & Horizontal alignment optimization \\
\hline Longitudinal alignment optimization & Route crossing optimization \\
\hline Project land occupation & Earthwork resource \\
\hline Centralized power supply in construction period & Low-dose crack-resistance cement stabilized macadam base \\
\hline Abandoned basalt water-stable macadam base & Warm-mix asphalt technology \\
\hline Prefabricated structure of bridge & Construction technology of environment-friendly bored pile \\
\hline Resource cyclic utilization of fly ash & Prestress intelligent tension system \\
\hline Full-circulation intelligent grouting system & Connection technology of thick rebar direct thread sleeve \\
\hline Economization of crash barrier & Selection of building materials \\
\hline Construction standardization & Low-carbon maintenance strategy \\
\hline Technical transformation of asphalt batching plant & Tunnel every-saving lighting technology \\
\hline All-weather rainy night reflector marking & Energy-saving direction sign \\
\hline Building of sound barrier & Multi-functional noise-reduction forest \\
\hline Green, ecological and environmental-protection project & Equipment and optimization of maintenance area \\
\hline Tunnel ventilation intelligent control system & ETC technology \\
\hline Expressway public service system & Pavement tracking and observation scheme \\
\hline Construction organization optimization scheme & $\begin{array}{c}\text { Construction period management, energy consumption statistics } \\
\text { and optimization }\end{array}$ \\
\hline Energy-saving operation and management system & Energy saving of vehicle trips \\
\hline $\begin{array}{c}\text { Operation energy consumption statistical monitoring } \\
\text { system }\end{array}$ & Science exhibition of green highway \\
\hline
\end{tabular}

\section{Guarantee measures of ecological highway construction}

\subsection{Strengthen system construction and energy consumption monitoring and evaluation}

The competent transportation department shall gradually perfect the energy saving and emission reduction system of transportation, study and put forward the green cycle low-carbon transportation development systematic frame, carry out prospective policy research on "green transport" leading transportation modernization, and evaluate the energy saving and emission reduction of transportation.

\subsection{Deepen special activities and strengthen the main role of enterprises}

The competent transportation department shall continuously promote modern engineering management, deeply carry out expressway construction standardization; promote legal land intensive use and saving of highway construction, promote recycle of pavement materials and tunnel energy-saving lighting of reconstruction and expansion project, strengthen technical research and development on the green cycle low-carbon transportation technologies such as large-scale comprehensive utilization of construction wastes, clean energy and renewable energy, carry out scientific and industrialization demonstration project, strengthen the promotion of scientific and technological achievements of energy saving and emission reduction, and carry out the promotion and application of energy-saving lighting technology and products of highway and tunnel.

\subsection{Strengthen publicity and exchange, and spread green traffic culture}

The competent transportation department shall 
continuously strengthen exchange and cooperation and utilize the multi/bilateral channel "bring in and go out", strengthen the exchange and cooperation of international organization, foreign government institutions, enterprises and research consultancy institution, widely utilize international resources and actively absorb and learn the international advanced experience, spread the green transport culture, study the green transport culture value system and construction evaluation system, and put forward the construction and spread scheme of green transport culture.

\section{Conclusion}

Ecological highway is an indispensable part in the green transportation system. Therefore, it is an intrinsic requirement for green transport development to clarify the idea of ecological highway construction and define the concept of ecological highway under the background of global warming, construct the ecological highway with the strategy of powerful transportation country, which lays the foundation for accelerating the reform of ecological civilization system in the new era, building a beautiful China, realizing the development of green transport and building a powerful transportation country

\section{References}

1. Chen Xiaoli. (2012) System Research on Key Technologies of Low-carbon Expressway System [J]. Chinese Journal of Underground Space and Engineering, (S1): 1358-1363.

2. Han Zhi, Fang Jianqin and Hong Weipeng (2010) Energy Saving Technology of Highway and Tunnel [M]. Beijing: China Communications Press.

3. Qin Xiaochun, Shen Yi, Shao Shegang and Huang Yujie (2010) Discussion on Key Technology and Application of Green Highway Construction under Low Carbon Concept [J]. Journal of Highway Communication Technology (application technology version), (10): 308-310.

4. Li Zhongshan (2009) Concluding Remarks on Promoting Ecological Highway Construction in China [J]. Communications Science and Technology Heilongjiang, (04): 141-142.

5. Ma Zhongnan and Gao Jiangang (2010) Discussion on Research System of Green Highway [J]. Journal of Highway Communication Technology, (08): 146-149.

6. Luo Jizhi (2009) Research on Mountainous Ecological Highway Construction based on Sustainable Development $[\mathrm{J}]$. Western China Communications Science \& Technology, (09): 142-148.

7. Wenbi Mao, Changqun Duan, et al. (2009) Highway Terrestrial Ecology [M]. China Communications Press, Beijing.

8. Tongfu Han (2011) Study on Highway Terrestrial Ecological Planning and Environmental impact
Assessment [D]. Tianjin University, Tianjin.

9. Shuguang $\mathrm{Wu}$ (2008) Study on Ecological Security Technology of Highway Construction [D]. Chang'an University, Xi'an.

10. Yi Shen \& Xiaolin Yan, (2009) Highway Terrestrial Ecological Engineering Technology [M].China Communications Press, Beijing.:3-6,20 